\title{
STUDIES ON THE CHEMICAL COMPOSITION OF PLANTS ASSOCIATED WITH FLOWER BUD FORMATION*\$
}

\author{
Y. Asami \\ and \\ H. Іто \\ Tokyo Imperial University \\ Faculty of Agriculture \\ Toliyo Imperial College
of Agriculture
}

Since the publication of the work by KraUS and KraYBILL, ${ }^{(5)}$ a great deal of interest has been centered on the study of the relative amounts of carbohydrates and nitrogen in relation to flower bud formation. The general belief that flower bud production is associated with increase in carbohydrate content seems to be supported by the results of various special practices such as ringing and defoliation. However, throughout the recent extensive works, the obtained results are not always the same, and the data which failed to show that the accumulation of carbohydrates favors flower bud formation, have been not rarely reported, ${ }^{(1)(3)(7)}$ showing that a complete understanding seems to be still very distant.

The studies reported in this paper represent the data obtained with the Japanese pear trees and the short-day plants.

\section{Materials and Methods}

\section{Experiments with Japanese Pear Shoots}

The vigorously growing shoots of fruit trees are sometimes pruned back in summer for the purpose of getting flower buds on them with a belief that an enough accumulation of carbohydrates would be induced in the pruned shoots through ehecking shoot growth. The authors, ${ }^{(2)}$ however, obtained in their experiment with pear shoots the result that summer pruning induced a rather increase of nitrogen. On the other hand, $\mathrm{K}_{\text {AWAGUCHI, }}{ }^{(4)}$ reported in his study with Japanese pear trees that the shoots which had been pruned back in May when they were growing vigorously, generally failed to form flower buds on them, but many of them headed at the end of June or in July when their elongation declined or nearly ended, produced flower buds.

In this study, the effects of early and late summer pruning on the

* A part of the investigations which are carried on with aid of the subsidiary foundation of the Memorial Service for the Tercentenary of the Tosho Shrine.

$\S$ This paper was presented before the extraordinary meeting of the Horticultural Association of Japan, held at the Hokkaido Imperial University on July 22 and 23, 1937.

Authors' acknowledgment. 'The writers wish to express their appreciation to Mr. M. Kawaguchi of the Kanagawa Agricultural Experiment Station for his help afforded during the experiment. 
chemical composition as related to flower bud production were examined in 1935. The shoots of the variety Chojuro were usad. The early heading which would be nearly uneffective for flower bud formation was conducted on May 27, and the late heading which would cause flower bud formation was done on July 4. Samples of each plot were taken for chemical analysis on July 20 when the flower bud differentiation was expected to occur in these shoots.

In Japanese pear trees, the shoots which have grown very vigorously form fow flower buds only on their apical portion, while those which have ceased the length growth early form many flower buds on them except on several basal nodes which bear leaf buds. The shoots of these two types we e chosen for study in 1936. Early in July, two groups of shoots, long growth and medium growth, were selected. The shoots of long growth were about $150 \mathrm{~cm}$. in length at that time and still growing. Such shoots are found mostly at the center of a horizontal overhead trellis, on which the tree-top of Japanese pear is trained, where the scaffold limbs are bent nearly at right angles. The shoot of medium growth were about $80 \mathrm{~cm}$. in length and had nearly completed length growth at that time. The half of shoots in each group was collected for chemical analysis on July 11, and the other half was remained on tree to examine flower bud formation. The shoots collected for chemical analysis were divided into three portions, top, middle and base, in the group of long growth, and two portions, top and base, in the group of medium growth. The pieces of about $12 \mathrm{~cm}$. long were eutted off from each portion and submitted to analysis.

\section{Experiments with Short-day Plants}

It will be very interesting to examine the effects of day-length on the chemical composition in shoit-day plants because short-day condition seems to be unfavorable for either carbohydrate accumulation or high carbohydrate-nitrogen ratio. NIGHTINGALE ${ }^{(6)}$ reported that carbohydrates accumulate in short-day, flowering salvia, but ARTHUR et al. ${ }^{(1)}$ found no relation between carbohydrate and nitrogen content and flowering in sa!via.

In this study, the effects of day-length upon carbohydrate and nitrogen content in relation to flower bud formation with cosmos and chrysanthemum, each of which is a typical short-day plant were examined. The potted plants in short-day plot were brought into a dark room at five o'clock each evening and kept there till eight o'clock in the following rnorning being exposed to a day-length shortened to 9 hours. To lengthen the photoperiod, 100-watt Mazda electric-light bulbs were suspended 2 feet above the top of plant and arranged at the rate of one bulb for 9 square feet of space. The lights were turned on at five o'clock and off 
at ten o'clock each night by the automatic time switch.

\section{Cosmos}

Treatments of photoperiod began ou July 13, 1935. The first sampling. for chemical analysis was made on August 11, when the flower buds began to be formed only in the short-day plot and no sign of flower bud formation was seen in both long-day and control plot. The second sampling was done on August 22, when the flower bud formation was initiated in the control plot, while the plants of long-day plot remained still in vegetative growth.

\section{Chrysanthemum}

A Japanese small flowered type was used in 1935. The treatment of photoperiod began on August 13. When the first sampling was made on September 11, the flower buds were formed in both short-day and control plot, while they were not yet found in long-day plot. On October 16, when the second sampling was made, the plants which had been transferred from long-day to short-day plot, formed flower buds, and they were compared with those which remained in long-day plot and formed yet no flower bud.

In 1936, a pink flowered variety Unaka was used. Photoperiodic treatment began on July 30, and the samples for chemical analysis were taken on August 13, when the flower bud formation began to occur in short-day plot, but not yet in control plot. Leaves, upper and lower portion of stems were submitted to analysis separately.

\section{Methods of Chemical Analysis}

The samples were eut with pruning shears as rapidly as possible into sections from one to three $\mathrm{mm}$. long, and preserved in 80 per cent alcohol until the extraction could be begun. For extraction the alcohol was filtered from the sample through a hardened paper. The residue wes dried and pulverized to pass a 100-mesh sieve, and was extracted thoroughly with alcohol. The estimation of polysaccharides and insoluble nitrogen was made upon the residue, and sugars and soluble nitrogen were estimated in the extract. The nitrogen was determined by the Kjeldahl me!hod, soluble nitrogen being modified as Ranker's proposition. Nitrate nitrogen was determined by the phenoldisulphonic acid method, amide and ammonia nitrogen were determined by the Van Slyke-Cullen method, and amino nitrogen was determined by the Van Slyke method. The carbohydrates were determined by the Bertland method. Starch was digested with Taka-diastase, and non-reducing sugars and polysaccharides was hydrolysed with hydrochloric acid as usually. 


\section{Results \\ Experiments with Japanese Pear Shoots}

The shoots which had been headed back on May 27, soon dereloped secondary growth and the flower buds were not generally formed on the primary growth portion. The shoots which had been pruned back on July 4, developed no secondary growth and a few apical buds on the headed portion differentiated into flower bud.

The chemical centents of the late headed shoots and the corresponding portion of early headed and unheaded shoots were shown in Table 1.

Table 1.-Chemical composition of Chojuro pear shoots.

Expressed on per Cent dry weight. Sampled on July 20, 1935.

\begin{tabular}{|c|c|c|c|}
\hline & $\begin{array}{c}\text { Upper portion } \\
\text { of shoots headed } \\
\text { on July } 4\end{array}$ & $\begin{array}{l}\text { Upper portion } \\
\text { (primary } \\
\text { grow th) of } \\
\text { shoots headed } \\
\text { on May } 27\end{array}$ & $\begin{array}{l}\text { Basal portion } \\
\text { of unheaded } \\
\text { (control) shoots }\end{array}$ \\
\hline Flower bud formation & $\begin{array}{c}\text { Flower buds } \\
\text { would be formed }\end{array}$ & $\begin{array}{l}\text { Flower buds } \\
\text { would not be } \\
\text { formed }\end{array}$ & $\begin{array}{l}\text { Flower buds } \\
\text { would not be } \\
\text { formed }\end{array}$ \\
\hline $\begin{array}{l}\text { Dry matter (per cent fresh } \\
\text { weight) } \ldots \ldots \ldots \ldots \ldots \ldots \ldots \ldots\end{array}$ & 51.55 & 45.77 & 44.53 \\
\hline Soluble solids $\ldots \ldots \ldots \ldots \ldots$ & 30 & 30 & 25 \\
\hline Insoluble solids $\ldots \ldots \ldots \ldots \ldots$ & 70 & 70 & 75 \\
\hline Soluble nitrogen $\ldots \ldots \ldots \ldots$ & 0.314 & 0.188 & 0.170 \\
\hline Amide and ammonia nitrogen .. & 0.0700 & 0.00532 & 0.00739 \\
\hline Amino nitrogen...$\ldots \ldots \ldots \ldots$ & 0.140 & 0.0591 & 0.047 \\
\hline Insoluble nitrogen $\ldots \ldots \ldots \ldots$ & 0.305 & 0.471 & 0.327 \\
\hline 'Total nitrogen $\ldots \ldots \ldots \ldots \ldots$ & 0.619 & 0.659 & 0.497 \\
\hline Reducing sugars $\ldots \ldots \ldots \ldots \ldots$ & 1.985 & 1.212 & 1.623 \\
\hline Non-reducing sugars $\ldots \ldots \ldots$. & 2.920 & 2.370 & 2.101 \\
\hline Total sugars $\ldots \ldots \ldots \ldots \ldots \ldots$ & 4.905 & 4.485 & 3.725 \\
\hline Water soluble polysaccharides.. & 0.406 & 0.447 & 0.392 \\
\hline Starch $\quad \ldots \ldots \ldots \ldots \ldots \ldots \ldots$ & 0.471 & 0.482 & 0.403 \\
\hline $\begin{array}{l}\text { Acid-hydrolysable polysaccha- } \\
\text { rides } \ldots \ldots \ldots \ldots \ldots \ldots \ldots \ldots\end{array}$ & 7.587 & 9.159 & 6.736 \\
\hline Total polysaccharides $\ldots \ldots \ldots$ & 8.464 & 10.088 & 7.531 \\
\hline Total carbohydrates ............ & 13.369 & 14.573 & 11.256 \\
\hline $\mathrm{C} \cdot \mathrm{N}$ ratio $\ldots \ldots \ldots \ldots \ldots \ldots \ldots$ & 21.60 & 22.11 & 22.64 \\
\hline
\end{tabular}

It is seen that either early or late summer heading brought about increase in the content of both total nitrogen and carbohydrate fractions. However, as flower bud formation occurred in the late heading plot and not in the early heading plot, when we make comparison of these two heading plots for chemical contents, the most striking feature of difference is the high content of soluble nitrogen in the lats heading plot, while 
there is no distinct difference in the content of sugars and starch, as well as in the $\mathrm{C}-\mathrm{N}$ ratio. The high content of soluble nitrogen seems, therefore, favorable for flower bud formation in this case.

In 1936, the shoots of two different growth types were studied. The distribution of flower buds on the shoots of two types was examined on I)ecember 7 , and is shown in Table 2.

Tabie 2.--Distribution of Flower buds on Chojero pear shoots. Examined on December 7, 1936.

\begin{tabular}{|c|c|c|c|c|c|c|c|c|c|}
\hline \multicolumn{5}{|c|}{ Shoots of long growth } & \multicolumn{5}{|c|}{ Shoots of medium growth } \\
\hline $\begin{array}{l}\text { Location of } \\
\text { flower buds* }\end{array}$ & 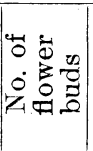 & 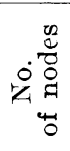 & 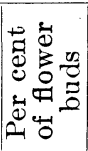 & 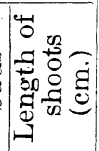 & $\begin{array}{l}\text { Location of } \\
\text { flower buds* }\end{array}$ & 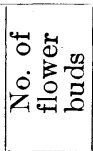 & 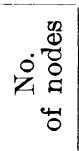 & 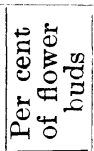 & 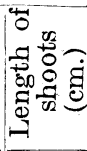 \\
\hline 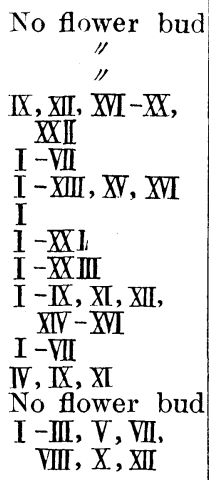 & $\begin{array}{r}0 \\
0 \\
0 \\
8 \\
7 \\
15 \\
1 \\
21 \\
23 \\
14 \\
7 \\
3 \\
0 \\
8\end{array}$ & $\begin{array}{l}38 \\
34 \\
35 \\
39 \\
31 \\
39 \\
38 \\
30 \\
30 \\
35 \\
33 \\
31 \\
30 \\
34\end{array}$ & $\begin{array}{r}20.5 \\
22.6 \\
38.4 \\
2.6 \\
70.0 \\
76.7 \\
40.0 \\
21.9 \\
9.7 \\
0 \\
23.5\end{array}$ & $\begin{array}{l}180 \\
170 \\
180 \\
173 \\
170 \\
175 \\
200 \\
150 \\
132 \\
193 \\
170 \\
144 \\
144 \\
170\end{array}$ & $\begin{array}{l}I-X V I \\
I-X \\
I-X I \\
I-\overline{Y I I} \\
I-X I V \\
I-X I I \\
I-X I \\
I-I X \\
I-V I I \\
I-X I V \\
I-X \\
I-X I I \\
I-X I I \\
I-X I \\
I-X Y I\end{array}$ & $\begin{array}{r}16 \\
10 \\
11 \\
8 \\
14 \\
16 \\
11 \\
9 \\
8 \\
14 \\
10 \\
16 \\
16 \\
15 \\
16\end{array}$ & $\begin{array}{l}18 \\
16 \\
17 \\
16 \\
15 \\
16 \\
17 \\
13 \\
20 \\
16 \\
20 \\
18 \\
19 \\
23 \\
21\end{array}$ & $\begin{array}{r}88.9 \\
62.5 \\
64.7 \\
50.0 \\
93.3 \\
100.0 \\
64.7 \\
69.2 \\
40.0 \\
87.5 \\
50.0 \\
88.9 \\
84.2 \\
65.2 \\
76.3\end{array}$ & \\
\hline Average & 7.6 & 34.1 & 23.3 & 168 & Average & 12.7 & 17.7 & 72.4 & 80 \\
\hline
\end{tabular}

* numbered basipetally

It is shown that on the shoots of long growth, the flower buds were formed only on apical portion and nearly no flower bud was produced on either middle or basal portion, and there were, moreover, in this group the shoots which borne no flower bud at all. On every shoot of medium growth, the flower buds were formed continuously from the apical end to a node lower than middle, remaining only basal portion in barren.

The chemical compositions examined with three portions in the shoots of long growth and two portions in the sboots of medium giowth are represented in Table 3 . 
Table 3.-Chemical composition of Chojuro pear shoot. Expressed on per cent dry weight. Sampled on July 11, 1936.

\begin{tabular}{|c|c|c|c|c|c|}
\hline & \multicolumn{3}{|c|}{ Shoots of long growth } & \multicolumn{2}{|c|}{ Shoo s of medium growth } \\
\hline & $\begin{array}{l}\text { Apical } \\
\text { portion }\end{array}$ & $\begin{array}{l}\text { Middle } \\
\text { portion }\end{array}$ & $\begin{array}{c}\text { Basal } \\
\text { portion }\end{array}$ & $\begin{array}{l}\text { Apical } \\
\text { portion }\end{array}$ & $\begin{array}{l}\text { Basal } \\
\text { portion }\end{array}$ \\
\hline $\begin{array}{l}\text { Flower bud } \\
\text { formation }\end{array}$ & $\begin{array}{c}\text { Flower buds } \\
\text { would be } \\
\text { formed }\end{array}$ & $\begin{array}{l}\text { Flower buds } \\
\text { would not } \\
\text { be formed }\end{array}$ & $\begin{array}{c}\text { Flower buds } \\
\text { would not } \\
\text { be formed }\end{array}$ & $\begin{array}{c}\text { Flower buds } \\
\text { would be } \\
\text { formed }\end{array}$ & $\begin{array}{l}\text { Flower buds } \\
\text { would not } \\
\text { be formerl }\end{array}$ \\
\hline $\begin{array}{l}\text { Dry matter (per } \\
\text { cent fresh weight) }\end{array}$ & 15 & 28 & 37 & 26 & 36 \\
\hline Soluble solids $\cdots$ & 38 & 16 & 10 & 22 & 11 \\
\hline Insoluble solids .. & 62 & 84 & 90 & 78 & 89 \\
\hline Soluble nitrogen .. & 0.240 & 0.141 & 0.102 & 0.389 & 0.150 \\
\hline Amide nitrogen .. & 0.00231 & 0.00151 & 0.00126 & 0.00312 & 0.00168 \\
\hline Ammonia nitrogen & 0.03709 & 0.02530 & 0.01568 & 0.05290 & 001695 \\
\hline Amino nitrogen .. & 0.165 & 0.102 & 0.068 & 0.227 & 0.105 \\
\hline Insoluble nitrogen & 1.210 & 0.625 & 0.483 & 0.914 & 0.541 \\
\hline Total nitrogen .... & 1.450 & 0.766 & 0.585 & 1.303 & 0.691 \\
\hline Reducing sugars .. & 2.048 & 1.169 & 0.764 & 1.017 & 0.927 \\
\hline $\begin{array}{l}\text { Non-reducing } \\
\quad \text { sugars } . . . . . .\end{array}$ & 4.250 & 1.618 & 0.991 & 2.868 & 0.927 \\
\hline Total sugars ...... & 6.298 & 2.787 & 1.755 & 3.885 & 1.854 \\
\hline $\begin{array}{l}\text { Water soluble } \\
\text { polysaccharides.. }\end{array}$ & 0.520 & 0.410 & 0.320 & 0.356 & 0.313 \\
\hline Starch $\ldots \ldots \ldots \ldots$ & 0.479 & 0.619 & 0.620 & 0.687 & 0.610 \\
\hline $\begin{array}{l}\text { Acid hydrolysable } \\
\text { polysaccharides }\end{array}$ & 4.169 & 3.116 & 3.200 & 3.090 & 3.200 \\
\hline $\begin{array}{l}\text { Total polysaccha- } \\
\text { rides............ }\end{array}$ & 5.108 & 4.145 & 4.140 & 4.133 & 4.123 \\
\hline $\begin{array}{c}\text { Total carbohydra- } \\
\text { tes } \ldots \ldots \ldots \cdots \cdots\end{array}$ & 11.406 & 6.932 & 5.895 & 8.018 & 5.977 \\
\hline $\mathrm{C}-\mathrm{N}$ ratio $\ldots \ldots$. & 7.860 & 9.050 & 10.077 & 6.153 & 8.650 \\
\hline
\end{tabular}

With the shoots of long growth, as well as of medium growth, it may be seen that there were evident increase in the content of nitrogen, both soluble and insoluble, and sugars, and decrease in the value of $\mathrm{C}-\mathrm{N}$ ratio toward the apical end. Though flower bud formation occurred on the apical portion of shoot of long growth, it occurred far more abundantly and constantly on the shoot of medium growth as shown in Table 2, and coinparing the apical portion of shoot of long growth and that of medium growth for the contents of nitrogen and carbohydrates, it is the content of soluble nitrogen which is obviously higher in the shoots of medium growth than in those of long growth. Therefore, in this case also, the soluble nitrogen content seems correlated positively with flower bud formation. 


\section{Experiments with Short-day Plants}

\section{Cosmos}

The results of chemical analysis with cosmos are shown in Table 4.

In the case in which the samples for chemical analysis were taken on $\Lambda$ ugust 11, it is shown that the contents of reducing sugars, total sugars and total carbohydrates are the lowest in the short-day plot and the highest in the long-day plot, indicating elearly that short-day condition actually decreased carbohydrate contents. On the contrary to carbohydrates, nitrogen, both soluble and insoluble, is the richest in the shortday plot. As the plants which formed flower buds were those under short days, high ecntent of nitrogen and low content of carbohydrates

TABle 4.--Chem:CaL Composition of cosmos.

Expressed on per cent dry weight. All aerial portion.

\begin{tabular}{|c|c|c|c|c|c|}
\hline \multirow{2}{*}{$\frac{\text { Sampling date }}{\text { Day-length }}$} & \multicolumn{3}{|c|}{ August 11} & \multicolumn{2}{|c|}{ August 22} \\
\hline & $\begin{array}{c}\text { Natural das } \\
\text { (control) }\end{array}$ & Short day & Long day & $\begin{array}{l}\text { Natural } \\
\text { day }\end{array}$ & Long day \\
\hline $\begin{array}{l}\text { Flower bud } \\
\text { formation }\end{array}$ & $\begin{array}{l}\text { No flower } \\
\text { buds }\end{array}$ & $\begin{array}{c}\text { Flower buds } \\
\text { were } \\
\text { producing }\end{array}$ & $\begin{array}{l}\text { No flower } \\
\text { buds }\end{array}$ & $\begin{array}{c}\text { Flower buds } \\
\text { were } \\
\text { producing }\end{array}$ & $\begin{array}{c}\text { No flower } \\
\text { buds }\end{array}$ \\
\hline $\begin{array}{l}\text { Green weight of a } \\
\text { plant (grams) } . .\end{array}$ & 49.0 & 38.3 & 39.0 & 40.0 & 37.1 \\
\hline $\begin{array}{l}\text { Dry matter (per } \\
\text { cent fresh weight) }\end{array}$ & 15.612 & 9.860 & 13.156 & 15.764 & 16.194 \\
\hline Soluble solids $\ldots$ & 7.381 & 3.338 & 5.067 & 4.806 & 4.535 \\
\hline Insoluble solids .. & 8.231 & 6.522 & 8.089 & 10.958 & 11.659 \\
\hline Soluble nitrogen . & 0.5275 & 1.8708 & 1.1575 & 0.6803 & 0.7682 \\
\hline Nitrate nitrogen & 0.1320 & 0.3549 & 0.1892 & 0.1452 & 0.1539 \\
\hline $\begin{array}{l}\text { Ammonia and } \\
\text { amide nitrogen } .\end{array}$ & 0.0224 & 00426 & 0.0319 & 0.0298 & 0.0278 \\
\hline Amino nitrogen .. & 0.0675 & 0.0821 & 0.6745 & 0.0571 & 0.0618 \\
\hline Insoluble nitrogen & 1.4544 & 2.6465 & 1.9205 & 2.0618 & 2.0029 \\
\hline Total nitrogen .... & 1.9819 & 4.5173 & 3.0780 & 2.7421 & 2.7711 \\
\hline Reducing sugars . & 4.2947 & 1.1157 & 8.2460 & 7.6080 & 8.7138 \\
\hline $\begin{array}{l}\text { Non-reducing } \\
\quad \text { sugars } \ldots . . . . .\end{array}$ & 0.7692 & 0.8416 & 1.6872 & 1.4265 & 1.7180 \\
\hline Total sugars ...... & 5.0639 & 1.9570 & 9.9332 & 9.0345 & 10.4318 \\
\hline $\operatorname{Starch} \ldots \ldots \ldots \ldots$ & 28396 & 3.5591 & 3.4656 & 4.0576 & 4.6103 \\
\hline $\begin{array}{l}\text { Acid hydrolysable } \\
\text { polysaccharides.. }\end{array}$ & 4.7883 & 44008 & 5.7076 & 8.3688 & 8.7323 \\
\hline $\begin{array}{l}\text { Total polysacchari- } \\
\text { des } \ldots . . . \ldots \ldots \ldots\end{array}$ & 7.6279 & 7.9599 & 9.1732 & 12.4264 & 13.3426 \\
\hline $\begin{array}{r}\text { Total carbohydra- } \\
\text { tes............... }\end{array}$ & 12.6918 & 9.9169 & 19.1064 & 21.4609 & 23.7744 \\
\hline $\mathrm{C}-\mathrm{N}$ ratio $\ldots \ldots$ & 6.41 & 2.05 & 6.21 & 7.82 & 8.59 \\
\hline
\end{tabular}


were associated with flower bud formation in this case.

With two plots, natural-day and long-day, of which the samples for chemical analysis were collected on August 22, when flower bud formation was occurring in the plants under natural days but did not occur in those under long days, the contents of nitrogenous fractions showed no distinct difference between two plots, and the carbohydrates were richer, though slightly but consistently, in the long-day plot. Therefore, the low content of carbohydrates was associated with flower bud formation in this case.

\section{Chrysanthemum}

With chrysanthemum, the samples for chemical analysis were taken on September 11 and October 16 in 1935, and August 13 in 1936, and the results are shown in Tables 5 and 6 .

The results obtained on September 11, 1935 show that all fractions of

Table 5.-Chemical composition of chrysanthemum.

Expressed ON PER CENT dRy Weight. EXPeriment IN 1935.

\begin{tabular}{|c|c|c|c|c|c|}
\hline \multirow{2}{*}{$\begin{array}{c}\text { Sampling date } \\
\text { Day-length }\end{array}$} & \multicolumn{3}{|c|}{ September 11} & \multicolumn{2}{|c|}{ October 16} \\
\hline & $\begin{array}{c}\text { Natural day } \\
\text { (control) }\end{array}$ & Short day & Long day & $\begin{array}{l}\text { Transferred } \\
\text { from long } \\
\text { day to } \\
\text { natural day }\end{array}$ & Long day \\
\hline $\begin{array}{l}\text { Flower bud } \\
\text { formation }\end{array}$ & $\begin{array}{c}\text { Flower buds } \\
\text { were } \\
\text { producing }\end{array}$ & $\begin{array}{c}\text { Flower buds } \\
\text { were } \\
\text { producing }\end{array}$ & $\begin{array}{l}\text { No flower } \\
\text { buds }\end{array}$ & $\begin{array}{c}\text { Flower buds } \\
\text { were } \\
\text { producing }\end{array}$ & $\begin{array}{l}\text { No fiower } \\
\text { buds }\end{array}$ \\
\hline $\begin{array}{l}\text { Green weight of a } \\
\text { plant (grams) }\end{array}$ & 35.5 & 26.3 & 28.8 & 61.8 & 56.8 \\
\hline $\begin{array}{l}\text { Dry matter (per } \\
\text { cent fresh weight) }\end{array}$ & 22.24 & 19.19 & 21.62 & 19.55 & 19.33 \\
\hline Soluble solids $\ldots$ & 9.27 & 8.07 & 8.93 & 6.47 & 6.95 \\
\hline Insoluble solids .. & 12.97 & 11.12 & 12.69 & 13.08 & 12.38 \\
\hline Soluble nitrogen . & 0.6660 & 0.9951 & 0.6436 & 0.5632 & 0.5067 \\
\hline Insoluble nitrogen & 2.0993 & 2.1528 & 2.1016 & 3.0336 & 2.5436 \\
\hline Total nitrogen .... & 2.7653 & 3.1479 & 2.7452 & 3.5968 & 3.0503 \\
\hline $\begin{array}{l}\text { Reducing sugars . } \\
\text { Non-reducing }\end{array}$ & 2.2950 & 1.6933 & 1.7826 & 1.6896 & 2.1352 \\
\hline sugars $\cdots \cdots \ldots$ & 2.6325 & 1.8443 & 2.7086 & 2.7392 & 4.7564 \\
\hline Total sugars ...... & 4.9275 & 3.5376 & 4.4912 & 4.4288 & 6.8916 \\
\hline Starch ............ & 1.1381 & 1.1004 & 1.1987 & 1.2052 & 1.1519 \\
\hline $\begin{array}{l}\text { Acid hydrolysable } \\
\text { polysaccharides. }\end{array}$ & 6.5952 & 4.8552 & 6.9450 & 6.3621 & 7.1687 \\
\hline $\begin{array}{c}\text { Total polysacchari- } \\
\text { des } \ldots \ldots \ldots \ldots \ldots\end{array}$ & 7.7333 & 5.9556 & 8.1437 & 7.5673 & 8.3206 \\
\hline 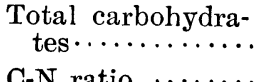 & 12.6608 & 9.4932 & 12.6349 & 11.9961 & 15.2122 \\
\hline C-N ratio $\ldots . . .$. & 4.58 & 3.01 & 4.60 & 3.33 & 4.98 \\
\hline
\end{tabular}


Table 6.-Chemical composition of Chrysanthemum.

EXPRESSED ON PER CENT DRY WEIGHT.

Experiment in 1936. Sampled on August 13.

\begin{tabular}{|c|c|c|c|c|c|c|}
\hline \multirow{3}{*}{$\frac{\text { Sampled portion }}{\text { Day-length }}$} & \multirow{2}{*}{\multicolumn{2}{|c|}{ Leaf blade }} & \multicolumn{4}{|c|}{ Stem } \\
\hline & & & \multicolumn{2}{|c|}{ Upper portion } & \multicolumn{2}{|c|}{ Lower portion } \\
\hline & $\begin{array}{l}\text { Natural } \\
\text { day }\end{array}$ & Short day & $\begin{array}{c}\text { Natural } \\
\text { day }\end{array}$ & Short day & $\begin{array}{l}\text { Natural } \\
\text { day }\end{array}$ & Short day \\
\hline $\begin{array}{l}\text { Flower bud } \\
\text { formation }\end{array}$ & $\begin{array}{c}\text { No flower } \\
\text { buds }\end{array}$ & $\left|\begin{array}{c}\text { Flower } \\
\text { buds were } \\
\text { producing }\end{array}\right|$ & $\begin{array}{c}\text { No flower } \\
\text { buds }\end{array}$ & \begin{tabular}{|l} 
Flower \\
buds were \\
producing
\end{tabular} & $\begin{array}{c}\text { No flower } \\
\text { buds }\end{array}$ & $\begin{array}{c}\text { Flower } \\
\text { buds were } \\
\text { producing }\end{array}$ \\
\hline $\begin{array}{l}\text { Dry matter (per } \\
\text { cent fresh weight) }\end{array}$ & 22.71 & 13.35 & 24.80 & 21.57 & 34.13 & 31.78 \\
\hline Soluble solids $\ldots$. & 3.65 & 1.74 & 5.91 & 4.86 & 5.49 & 4.57 \\
\hline Insoluble solids $\ldots$ & 19.06 & 11.61 & 18.89 & 16.71 & 28.64 & 27.21 \\
\hline Soluble nitrogen .. & 0.1761 & 0.2996 & 0.2734 & 0.3598 & 0.2151 & 0.2354 \\
\hline Insoluble nitrogen & 3.2057 & 3.845 & 0.6996 & 0.7418 & 0.4251 & 0.4579 \\
\hline Total nitrogen .... & 3.3818 & 4.1447 & 0.9730 & 1.1016 & 0.6402 & 0.6933 \\
\hline Reducing sugars .. & 2.3145 & 2.6219 & 2.3184 & 2.3180 & 0.9889 & 0.7868 \\
\hline $\begin{array}{l}\text { Non-reducing } \\
\quad \text { sugars } \ldots . . . . . .\end{array}$ & 1.8575 & 0.9738 & 7.6406 & 7.1858 & 5.2008 & 4.6733 \\
\hline Total sugars.... & 4.1720 & 3.5957 & 9.9590 & 9.5038 & 6.1897 & 5.4601 \\
\hline $\begin{array}{l}\text { Water-soluble } \\
\text { polysaccharides. }\end{array}$ & 1.0731 & 1.0869 & 0.5524 & 0.3741 & 0.6505 & 0.6272 \\
\hline $\operatorname{Starch} \ldots \ldots \ldots \ldots$ & 3.4281 & 1.5079 & 1.3209 & 0.8957 & 0.4632 & 0.2159 \\
\hline $\begin{array}{l}\text { Acid hydrolysable } \\
\text { polysaccharides.. }\end{array}$ & 16.4787 & 15.6892 & 12.6585 & 12.8130 & 157760 & 15.0710 \\
\hline $\begin{array}{l}\text { Total polysaccha- } \\
\text { rides } \ldots . \cdots \cdots \cdots\end{array}$ & 20.9799 & 18.2840 & 14.5318 & 14.0828 & 16.8897 & 15.9141 \\
\hline $\begin{array}{r}\text { Total carbohydra- } \\
\text { tes } . . . . . . . . . . .\end{array}$ & 25.1919 & 21.8797 & 24.4908 & 23.5866 & 23.0794 & 21.3742 \\
\hline $\mathrm{C}-\mathrm{N}$ ratio $\ldots \ldots$ & 7.44 & 5.28 & 25.17 & 21.41 & 31.47 & 30.83 \\
\hline
\end{tabular}

carbohydrates were the lowest in content in the short-day plot though there. was no consistent difference between the natural-day and the longday plot, and the nitrogen was the richest in the short-day plot, but the natural-day and the long-day plot were not noticeably different in the nitrogen contents. In this case in which flower bud formation occurred not only in the short-day plot but also in the natural-day plot, any association can not be seen between flower bud formation and chemical contents.

The results obtained on October 16, 1935 show that transferring from the long-day condition to the short-day condition, through which flower bud formation was induced, decreased clearly the contents of carbohydrates and increased, though not greatly, the nitrogen contents. The flower bud formation in this case was, therefore, associated with high content 
of nitrogen and low content of carbohydrates.

The results which were obtained on August 13, 1936 and are shown in Table 6, indicate that the contents of carbohydrates were decreased by short days, and that the flower bud formation was correlated also in this case with higher content of nitrogen and lower content of carbuhydrates.

From the above mentioned results obtained with the short-day plants, it may be seen that the contents of carbohydrates were decreased by short days, and the flower bud formation was associated with not increase but decrease in the contents of carbohydrates with exception of one case, and it was associated at the same time with increase in nitrogen content, though it was not limited to soluble nitrogen, in 3 cases out of 5 cases ex:mined.

\section{Summary}

1. In the results of this study the carbohydrate-nitrogen ratios have not proved of special significance in flower bud formation.

2. In the comparison of chemical composition between early summer headed and late summer headed shoots and also between shoots of long growth and those of medium growth of the Chojuro pear trees, the high content of soluble nitrogen was associated with flower bud formation.

3. The results of the study with short-day plants such as cosmos and chrysanthemum show that the content of carbohydrates was decreased by short days and flower bud formation in these plants was, therefore, associated with decrease in carbohydrates on the contrary to the general belief that the accumulation of carbohydrates favors flower bud production.

4. Within the limits of the data obtained in this study, high content of nitrogen, though not in every case, but on the whole, seems farorable for flower bud formation.

\section{Literature Cited}

(1) Arthur, J. M., Guthrie, G. D., and Newell, J. M. : Some effects of artifcial climates on the growth and chemical composition of plants. Amer. Journ. Bot. 17 : 416-482. 1930.

(2) Asami, Y., and Iто, H.: A preliminary report on the effects of summer pinching upon the carbohydrate and nitrogen contents of the Japanese pear shoots. Journ. Hort. Assoc. Japan IV, 2: 59-65. 1933.

(3) Heinicke, A. J.: Composition of fruit-bud and spur tissues of Wealthy apples under different condition of nutrition. Proc. Amer. Soc. Hort. Sci. 27 (1930) : 190-198. 1931 .

(4) Kawaguchi, M.: Effects of summer pruning on development of secondary growth and flower bud formation of the Japanese pear shoots (in Japanese). Agriculture and Horticulture 4:563-569. 1929.

(5) Kraus, E. J., and Kraybill, H. R.: Vegetation and reproduction with special reference to the tomato. Oregon Agr. Exp. Sta. Bull. 149. 1918.

(6) Nightingale, G. T.: The chemical composition of p'ants in relation to photoperiodic changes. Wisconsin Agr. Exp. Sta. Bull. 74. 1927.

(7) Potter, G. F., and Prilitips, T. G.: Composition and fruit bud formation in non-bearing spurs of the Baldwin apple. New Hampshire Agr. Exp. Sta Tech. Bull. 42. 1930. 